\title{
Group differences in adult simple arithmetic: Good retrievers, not-so-good retrievers, and perfectionists
}

\author{
STEVEN A. HECHT \\ Florida Atlantic University, Davie, Florida
}

\begin{abstract}
We used the choice/no-choice methodology in two experiments to examine patterns of strategy selection and execution in groups of undergraduates. Comparisons between choice and no-choice trials revealed three groups. Some participants (good retrievers) were consistently able to use retrieval to solve almost all arithmetic problems. Other participants (perfectionists) successfully used retrieval substantially less often in choice-allowed trials than when strategy choices were prohibited. Not-sogood retrievers retrieved correct answers less often than the other participants in both the choiceallowed and no-choice conditions. No group differences emerged with respect to time needed to search and access answers from long-term memory; however, not-so-good retrievers were consistently slower than the other subgroups at executing fact-retrieval processes that are peripheral to memory search and access. Theoretical models of simple arithmetic, such as the Strategy Choice and Discovery Simulation (Shrager \& Siegler, 1998), should be updated to include the existence of both perfectionist and not-so-good retriever adults.
\end{abstract}

Until recently, process models of simple arithmetic (e.g., $4+3$ or $5 \times 2$ ) in adults focused almost exclusively on retrieval processes (see, e.g., Ashcraft, 1995; Widaman, Geary, Cormier, \& Little, 1989). Indeed, it was largely assumed that adults relied on retrieval of answers from long-term memory to solve simple arithmetic addition and multiplication problems (e.g., $4+3$ and $4 \times 3$; Hecht, 1999). However, there is converging evidence that a substantial number of undergraduates actually use strategies that are slower and more error prone than retrieval to solve some simple arithmetic problems (see, e.g., Campbell \& Xue, 2001; Hecht, 1999, 2003; LeFevre, Sadesky, \& Bisanz, 1996). The aim of this study was to further our understanding of why some adults use multiple strategies in simple arithmetic.

We interpreted the present research within the context of a general model of cognitive development that has been used to explain simple arithmetic performance in children: the Strategy Choice and Discovery Simulation (SCADS; Shrager \& Siegler, 1998). According to the SCADS, when a person attempts to solve a simple arithmetic problem, representations of the problem, its candidate answers, and codes corresponding to characteristics of candidate strategies (speed and efficacy) are activated. The strategy that receives the most activation from the presented simple arithmetic problem is chosen first. Retrieval is usually used when the problem is highly associated with the correct answer representation in long-term memory

Correspondence concerning this article should be addressed to S. A. Hecht, Psychology Department, Florida Atlantic University, 2912 College Avenue, Davie, FL 33314 (e-mail: shecht@fau.edu). rather than with incorrect answers. (This is referred to as a "peaked" distribution.) Retrieval is predicted to be used almost exclusively because of the amount of cumulative problem-solving experience adults have (Siegler \& Shipley, 1995).

A modified version of the SCADS could explain reported multiple-strategy use in adults. Selection of more time-consuming and error-prone nonretrieval strategies may sometimes reflect an adaptive strategy choice for some people (Geary \& Wiley, 1991; Hecht, 1999). That is, some adults (not-so-good retrievers) may simply be unable to implement retrieval effectively for some simple arithmetic problems. The SCADS assumes that a particular procedure tends to be selected when that procedure can be executed efficiently (i.e., with sufficient speed and efficacy). In Shrager and Siegler's (1998) model, the associative strength between a problem and a nonretrieval procedure could become stronger than the link between the problem and its correct answer (LeFevre, Sadesky, \& Bisanz, 1996). After this strong link between a problem and a nonretrieval strategy has been established, some adults may continue to use the nonretrieval strategy for the problem, rather than shifting to a retrieval strategy (Geary \& Wiley, 1991; Hecht, 1999).

Multiple-procedure usage, rather than exclusive use of retrieval, may also reflect nonadaptive strategy choices for some adults (cf. Hecht, 1999; Touron \& Hertzog, 2004). Within the context of the SCADS, some students may choose a nonretrieval strategy, even when their distribution of associations for a particular problem is sufficiently peaked. This perfectionist pattern of responding has been identified in children (Kerkman \& Siegler, 1993; Siegler, 1988a). So-called perfectionist children are very accu- 
rate when using the retrieval strategy, yet continue to use nonretrieval strategies to solve many simple arithmetic problems (Siegler, 1988a). Adults rarely make retrieval errors, yet some solve problems via nonretrieval strategies, suggesting the existence of perfectionist adults (Geary \& Wiley, 1991).

\section{Overview of the Present Study}

The purpose of this study was to answer two research questions concerning strategy selection and execution processes in adult simple arithmetic. The first question was whether perfectionists, not-so-good retrievers, and people who consistently solve arithmetic problems via retrieval (i.e., good retrievers) could be identified in the present adult samples. As part of our examination, we considered the nature of strategy execution in these subgroups of adults. The second research question was whether subgroup differences would emerge on measures of working memory and general math achievement.

We addressed the first research question using the choice/no-choice method (see, e.g., Jordan, Hanich, \& Kaplan, 2003; Siegler \& Lemaire, 1997). Specifically, the mean percentage of trials in which adults successfully used retrieval when allowed to choose a strategy (choice condition) was compared with the mean percentage of correct retrieval use when nonretrieval strategy selection was prohibited (no-choice condition). In the no-choice condition, adults were instructed to use only retrieval to solve arithmetic problems (Rittle-Johnson \& Siegler, 1999). If an answer could not be retrieved in the no-choice condition, then the person would either not state an answer or ignore the instructions and resort to a nonretrieval strategy. Within the context of the SCADS, the no-choice condition allowed an estimation of people's distributions of associative strengths that was minimally influenced by decision processes (Siegler \& Lemaire, 1997).

By comparing each adult's mean use of retrieval in the no-choice and choice conditions, we tested for the emergence of three specific subgroups of adults, as follows: Not-so-good retrievers were individuals who were unable to retrieve the correct answers to a substantial proportion of simple arithmetic problems in both the choice and no-choice conditions. Perfectionists were adults who retrieved correctly on virtually every trial in the no-choice condition, yet chose nonretrieval on a substantial number of items in the choice condition. Good retrievers were participants who successfully used retrieval on the vast majority of trials in both the no-choice and choice conditions.

Execution of strategies refers to processes that occur when a particular strategy is used (Hecht, 2002). Consistent with the SCADS, even for problems that are answered via retrieval, the relative strength of the link between problems and correct answers may be weaker in not-so-good retriever adults than in other participants. Thus, these individuals should engage in memory-search and -access processes more slowly than those in the perfectionist and good retriever subgroups. An alternative prediction would be equivalent rates of fact-retrieval processes in the three adult subgroups. Memory retrieval in simple arithme- tic appears to be at, or very close to, asymptote by high school in students schooled in North America (Geary \& Wiley, 1991; Kail, 1988; Widaman, Little, Geary, \& Cormier, 1992). Indeed, even extended practice does not appear to increase the speed with which memory-search and -access processes in simple arithmetic occur in adults (Frensch \& Geary, 1993).

The second research question addressed in this study was whether or not subgroup differences would emerge on measures of working memory and mathematical-computation achievement. Working memory refers to how well a person can maintain and simultaneously process information in his or her focus of attention (Baddeley, 1992; Hecht, 2003). Working memory is typically measured with the counting-span task (see, e.g., Hitch \& McAuley, 1991). One learning mechanism assumed by the SCADS is that each time an answer is stated, the link between that answer and the simple arithmetic problem is strengthened. The degree of strengthening may be constrained by how well the individual can simultaneously represent and process the terms (e.g., via counting) and the generated answer in working memory (Geary, 1993).

Those with poor working memory skills may not readily form problem-answer associations. Moreover, for these individuals, strong links between problems and nonretrieval strategies are likely to be formed (Geary, 1993). Thus, not-so-good retriever adults may show poorer working memory and greater reliance on nonretrieval strategies than do good retrievers and perfectionists. Regarding solution times, Geary and Widaman (1992) reported a moderate correlation between adults' working memory and a variable representing both the speed of executing arithmetical memory-search and -access processes and the time to perform the carry operation in complex multidigit arithmetic $(r x y=-.35)$.

It would seem to be common sense that people who master more complex mathematical procedures (e.g., fractions, algebra, calculus) should show more efficient solving of relatively basic arithmetic facts (see, e.g., Gagne, 1983; Goldman, Mertz, \& Pellegrino, 1989; Hecht, 1998). People with efficient arithmetic skills can devote limited attentional resources to other processes involved in more complex mathematical procedures. Hecht (1999) employed a standardized test of participants' mastery of mathematicalcomputation skills (i.e., the Calculation subtest from the revised Woodcock-Johnson Psycho-Educational BatteryRevised; Woodcock \& Johnson, 1989). Performance on this standardized math test correlated only marginally with adults' frequency of using retrieval $(r x y=.21, p<.10)$. It is possible that the presence of perfectionists in studies of simple arithmetic may reduce the simple linear associations between the percentage of retrieval usage and math achievement.

\section{METHOD}

In the first experiment, undergraduates were administered tests of their simple arithmetic (addition and multiplication), working memory, and standardized math-computation achievement. These 
assessments were given in one session. The second experiment included the same measures. Two potential factors that limited the assuredness with which we could draw conclusions from Experiment 1 were addressed in Experiment 2. First, the present version of the SCADS predicts that typical college students should use retrieval virtually all of the time, so it seemed desirable to replicate the results with a different sample of undergraduates. With Experiment 2, we also wanted to preclude any potential influences of carryover effects from the first to the second administration of the simple arithmetic tasks. Accordingly, Experiment 2 involved two sessions; the participants were given the second administration of the arithmetic tasks after at least 1 week had elapsed.

\section{Participants: Experiments 1 and 2}

In Experiment 1, 78 undergraduate psychology students at Florida Atlantic University participated for course extra credit. In Experiment 2, 85 new undergraduate psychology students at Florida Atlantic University participated for course extra credit.

\section{Materials: Experiments 1 and 2}

1. Simple arithmetic: Addition. The participants were given problems drawn from one of two stimulus sets. None of the problems involved two equal numbers (e.g., $4+4$ or $5 \times 5$ ) or included a 1 or 0 as a term in the problem. Both sets included one of two possible combinations of single-digit integers, from $2+3$ to $8+9(n=28)$. Approximately half of the sample was given Stimulus Set A. The operand order for any given problem in Stimulus Set A was the opposite of that given in Stimulus Set B. For example, Stimulus Set A included the lowest term in the first position for the simple arithmetic problem with terms of 2 and 3 (i.e., $2+3$ ), whereas Stimulus Set $B$ included the highest term in the first position (i.e., $3+2$ ). For each set, half of the items were presented with the minimum operand to the left of the operation sign, and the other problems were given with the minimum term to the right of the operation sign. Problems were presented horizontally in white characters against a black background, in standard mathematical form (e.g., $2+9=$ ). The order in which the stimuli were presented was randomized independently for each participant.

2. Simple arithmetic: Multiplication. This task was identical to the previous task, except that the stimuli were multiplication simple arithmetic problems.

3. Standardized math computation. The participants solved various kinds of math-computation problems (e.g., multidigit division, fraction computation, algebra) at their own pace. This task was the Calculation subtest from the standardized Woodcock-Johnson Psycho-Educational Battery-Revised (1989), which provides an estimate of the total number of computation algorithms mastered by a student. The age-based standardized score is scaled to have a mean of 100 and a standard deviation of 15 . There are 58 items on this test.

4. Working memory. We used the counting-span task, which is typically used to measure working memory (Baddeley, 1992; Hitch \& McAuley, 1991). For each trial, the participants were first shown a screen with circles and squares and asked to state the number of circles. Next, the participants were shown additional screens; for each screen, the participants again indicated aloud the number of circles. After the students had been presented a certain number of screens, they were asked to indicate the number of circles counted from each screen. The participants were given two sets of trials involving two, three, four, five, six, and seven screens (six trials altogether). Accuracy of recalled counts was recorded. The most possible correct answers on this task was 12 .

5. Number-naming speed. The participants were presented a card with six rows of five single digits per row and instructed to name the digits as quickly as possible. Two trials were given. This task has been used extensively to measure general speed of retrieving familiar name codes in children (see, e.g., Wagner, Torgesen, \& Rashotte, 1994). Performance was measured in terms of the number of items named per second.

\section{Procedure: Experiment 1}

Each participant was tested individually in a quiet room. Each session lasted no more than approximately $1 \mathrm{~h}$. All of the participants were given the simple arithmetic addition and multiplication tasks first. At the beginning of the session, some participants were given the simple arithmetic tasks under the strategy choice-allowed condition $(n=35)$, whereas others received the no-choice (i.e., use retrieval only) instructions $(n=43)$. Next, the students were administered the working memory, computation, and naming-speed tasks in a random order. Finally, all of the participants were again administered the simple arithmetic tasks. The participants who first solved the problems under the choice-allowed condition were given the second administration of the arithmetic problems with no-choice instructions, or vice versa.

The general procedure for all arithmetic trials was as follows. At the beginning of the task, the participants were told that they would be seeing simple arithmetic problems on the computer screen and would be solving the problems as quickly as possible. Just before each trial began, the students were presented a dollar sign in the middle of the screen. The screen was cleared for $500 \mathrm{msec}$, and then the simple arithmetic problem was presented in the middle of the screen. The stimulus remained displayed until either $5,000 \mathrm{msec}$ had elapsed or the participant had uttered an answer into a headset microphone. After each trial, the experimenter wrote verbatim the participant's verbal descriptions of strategy usage for that problem. The experimenter then pressed the space bar to initiate the next trial. The first three trials were warm-up trials.

The specific instructions for the no-choice addition trials were as follows. After the first problem had been answered, the experimenter read this statement aloud while the participant followed along on the monitor:

One way that we can solve math facts is by simply remembering the answer by using retrieval. The answer just sort of pops into your head. This test is measuring how well you can remember the answers to math facts. It is important that you figure out the answer to the problem by using retrieval only. Please give your best guess if you don't remember the answer. Remember to say the answer as fast as you can.

Next, the following instructions were displayed on the computer screen, and the experimenter read them aloud while the participant followed along:

Remember that this is a test for retrieval only. If you do not know the answer, just give your best guess. Please remember to use retrieval.

For subsequent trials, if a nonretrieval strategy was used, the experimenter reminded the undergraduate to use retrieval. The procedure for no-choice multiplication trials was identical, except that "multiplication" was substituted for "addition" in the instructions.

The specific instructions for the choice-allowed addition trials were as follows. After the first problem had been answered, the participant was given the following instructions, taken verbatim from Kirk and Ashcraft (2001). These instructions are biased away from the use of nonretrieval strategies. Thus, the instructions do not cause demand-induced biases that encourage participants to choose nonretrieval strategies (Kirk \& Ashcraft, 2001). The instructions were displayed on the computer screen, and the experimenter read them aloud while the participant followed along:

What do people do when asked to add $4+5$ ? You could just remember the answer, 9. It just sort of pops into your head. That is how adults usually solve problems of this size. Or you might solve the problem by using a strategy. Of course, adults almost always just remember the answer to problems of this size, and we would like to know more about that process.

The procedure for choice-allowed multiplication trials was identical to that for choice-allowed addition, except that "multiplication" was substituted for "addition" in the instructions.

Coding of solution procedures. The verbal protocols for the strategy choice-allowed trials were coded based on categories used 
by LeFevre and her colleagues (LeFevre, Bisanz, et al., 1996; LeFevre, Sadesky, \& Bisanz, 1996). For addition, the following categories of responses were coded. Responses were coded as retrieval when the participants claimed that they remembered the answer. Decomposition procedures involved the use of a known arithmetic fact to find an answer. For example, the student might solve the problem $6+7$ by finding the solution to $6+6$ and then adding 1 to the answer. Min counting involved counting from the larger addend a number of increments equal to the smaller addend (e.g., "5 plus 2 equals 5, 6, 7; 7 is the answer"). Finally, an other category was used to code responses that were not consistent with the other substantive strategies for addition (e.g., memory aids such as visualizing the answer in a math fact table or using one's fingers). For multiplication trials, as with addition problems, responses were coded as "retrieval," "decomposition," or "other." For no-choice trials, the categories of procedures were "retrieval," "backup strategies," and "don't know." Backup strategies involve using any reconstructive procedure for figuring out the answer to the problem (Siegler, 1988a). Don't know responses occurred when the participant was unable to retrieve an answer within the 5-sec time limit.

\section{Procedure: Experiment 2}

The procedure was identical to that followed in the first experiment, with the following exceptions. The first session involved solving simple arithmetic problems under either choice-allowed or no-choice instructions. Next, the participants were given two of the three other tasks (i.e., number-naming speed and working memory), the order of which was chosen randomly for each person. For the second session, the participants were first administered the simple arithmetic problems (using the instructions not used in the first session). Some of the participants were given the simple arithmetic problems under choice-allowed instructions in the first session $(n=$ 42 ), whereas the other participants received the no-choice instructions in Session $1(n=43)$. The participants performed the standardized math-computation task after all the other tasks had been administered. Between 7 and 10 days elapsed between the first and second sessions.

\section{RESULTS}

\section{Selection of Strategies and Accuracy}

For each participant, the frequency of procedure use, error rate, and mean latencies for each substantive procedure category were determined. These values are reported in Table 1, segregated by operation (addition or multiplication) and choice condition (choice-allowed vs. no-choice). In the present Experiment 1, the participants used retrieval in $74 \%$ and $77 \%$ of the choice trials for addition and multiplication, respectively. In the no-choice condition, the participants used retrieval significantly more often for both addition and multiplication than when they were allowed to choose a strategy-98\% and $97 \%$ of the trials, respectively $\left[F_{\mathrm{s}}(1,77)=115.76\right.$ and 93.32 , $p \mathrm{~s}<.001$ ]. In Experiment 2, a similar pattern of results occurred, with significantly more use of retrieval for both addition and multiplication on no-choice trials (i.e., 94\% and $96 \%$, respectively) than on choice-allowed trials (i.e., $79 \%$ and $85 \%)[F \mathrm{~s}(1,84)=61.82$ and 40.73 , respectively, $p \mathrm{~s}<.001]$.

Accuracy levels for retrieval trials were impaired when the students were not given a choice, in comparison with the choice-allowed condition. For Experiment 1, addition and multiplication retrieval accuracy for choiceallowed trials was $98 \%$ and $99 \%$ correct, respectively.
These values significantly dropped to $93 \%$ and $94 \%$ correct, respectively $\left[F_{\mathrm{s}}(1,77)=7.85\right.$ and $10.96, p \mathrm{~s}<.01$, respectively]. Similarly, for Experiment 2, accuracy for retrieval trials was significantly reduced, by $4 \%$ and $3 \%$ for addition and multiplication, respectively $[F \mathrm{~s}(1,84)=$ 12.64 and $10.36, p \mathrm{~s}<.001$, respectively]. This reduction in accuracy levels is consistent with other research showing that prohibiting people from choosing strategies can impair accuracy when compared with allowing strategies to be freely selected (see, e.g., Siegler \& Lemaire, 1997).

\section{Individual Difference Groups}

Strategy selection. The first question was whether multiple-strategy usage by some adults in simple arithmetic reflects the presence of not-so-good retrievers and/or of perfectionists. To that end, we examined individual patterns of strategy choices in both the choice-allowed and no-choice conditions. Following previous work with children as participants (Kerkman \& Siegler, 1993; Siegler, 1988a), we used cluster analysis (a nonhierarchical $k$-means clustering algorithm based on Euclidean distances) to identify three relatively homogeneous groups of cases, based on selected variables. For each person, the following four variables were entered into the cluster analysis routine: (1) mean percentage of choice-allowed addition trials correctly solved via retrieval, (2) mean percentage of choice-allowed multiplication trials successfully solved via retrieval, (3) mean percentage of no-choice addition trials solved correctly via retrieval, and (4) mean percentage of no-choice multiplication trials successfully solved via retrieval.

The results from the three-cluster solution are reported in Table 2, segregated by experiment. As has been reported in studies with children (e.g., Siegler, 1988a), the threecluster solution identified in the adult sample conformed to characterizations of good retrievers, not-so-good retrievers, and perfectionists. For Experiment 1, there were 36 adults in the good-retriever cluster, 16 in the not-sogood-retriever cluster, and 26 in the perfectionist cluster. For Experiment 2, there were 45, 21, and 19 participants in these clusters, respectively.

For each group, the mean percentages of problems that were correctly retrieved were compared between the choice-allowed and no-choice conditions. In both Experiments 1 and 2, for not-so-good retrievers, no significant differences in mean successful-retrieval frequency were found between the choice-allowed and no-choice conditions for addition and multiplication problems $(F \mathbf{S}<1)$. For good retrievers, successful retrieval usage increased by approximately $9 \%$ and $4 \%$ for addition and multiplication in Experiment $1[F \mathrm{~s}(1,35)=32.47$ and 10.17, $p \mathrm{~s}<$ $.01]$ and $4 \%$ and $2 \%$ in Experiment $2\left[F_{\mathrm{s}}(1,44)=8.1\right.$ and $3.4, p<.01$ and $p<.10$, respectively]. Thus, in general, even good retrievers tended to use retrieval slightly less often than they could have.

Perfectionists successfully used retrieval substantially more often when they were not given a choice than when they were allowed to choose strategies, for both addition and multiplication, in Experiments $1[F \mathrm{~s}(1,25)=294.50$ 
Table 1

Selection of Procedures and Latencies, Segregated by

Operation and Instructions for Both Experiments

\begin{tabular}{|c|c|c|c|c|c|}
\hline Procedure & Use & Sample & Range in Use & RT (msec) & Accuracy \\
\hline \multicolumn{6}{|c|}{ Experiment 1} \\
\hline \multicolumn{6}{|c|}{ Addition: Choice allowed } \\
\hline Retrieval & .74 & 1.0 & $.32-1.0$ & 860 & .98 \\
\hline Decomposition & .13 & .73 & $.00-.57$ & 1,452 & .96 \\
\hline Min counting & .10 & .60 & $.00-.68$ & 1,533 & .92 \\
\hline Miscellaneous & .02 & .30 & $.00-.25$ & 1,379 & .72 \\
\hline Total errors & .03 & .53 & $.00-.18$ & 1,245 & - \\
\hline \multicolumn{6}{|l|}{ Addition: No choice } \\
\hline Retrieval & .98 & 1.0 & $.61-1.0$ & 889 & .93 \\
\hline Backup & .01 & .21 & $.00-.11$ & 1,113 & .44 \\
\hline Don’t know & .01 & .27 & $.00-.15$ & - & - \\
\hline \multicolumn{6}{|c|}{ Multiplication: Choice allowed } \\
\hline Retrieval & .77 & 1.0 & $.32-1.0$ & 1,058 & .99 \\
\hline Decomposition & .15 & .67 & $.00-.57$ & 1,920 & .89 \\
\hline Miscellaneous & .08 & .60 & $.00-.39$ & 1,952 & .64 \\
\hline Total errors & .05 & .54 & $.00-.29$ & - & - \\
\hline \multicolumn{6}{|c|}{ Multiplication: No choice } \\
\hline Retrieval & .97 & 1.0 & $.25-1.0$ & 947 & .94 \\
\hline Backup & .01 & .15 & $.00-.11$ & 1,242 & .83 \\
\hline Don't know & .02 & .33 & $.00-.14$ & - & - \\
\hline \multicolumn{6}{|c|}{ Experiment 2} \\
\hline \multicolumn{6}{|c|}{ Addition: Choice allowed } \\
\hline Retrieval & .79 & 1.0 & $.38-1.0$ & 865 & .98 \\
\hline Decomposition & .11 & .62 & $.00-.43$ & 1,652 & .87 \\
\hline Min counting & .08 & .54 & $.00-.46$ & 1,828 & .96 \\
\hline Miscellaneous & .02 & .21 & $.00-.22$ & 1,567 & .85 \\
\hline Total errors & .03 & .48 & $.00-.21$ & - & - \\
\hline \multicolumn{6}{|l|}{ Addition: No choice } \\
\hline Retrieval & .94 & 1.0 & $.64-1.0$ & 951 & .94 \\
\hline Backup & .04 & .19 & $.04-.19$ & 1,519 & .95 \\
\hline Don't know & .02 & .46 & $.04-.36$ & - & - \\
\hline \multicolumn{6}{|c|}{ Multiplication: Choice allowed } \\
\hline Retrieval & .85 & 1.0 & $.43-1.0$ & 1,147 & .98 \\
\hline Decomposition & .09 & .66 & $.00-.25$ & 2,103 & .87 \\
\hline Miscellaneous & .09 & .59 & $.00-.48$ & 1,887 & .63 \\
\hline Errors & .05 & .53 & $.00-.33$ & - & - \\
\hline \multicolumn{6}{|c|}{ Multiplication: No choice } \\
\hline Retrieval & .96 & 1.0 & $.79-1.0$ & 1,036 & .95 \\
\hline Backup & .02 & .29 & $.04-.08$ & 1,565 & .91 \\
\hline Don't know & .02 & .33 & $.04-.18$ & - & - \\
\hline
\end{tabular}

Note- Use, average proportion of trials in which individual participants used that strategy; Sample, proportion of participants who used that strategy at least once; Range in Use, range of proportions for trials in which individual participants used that strategy; RT, average of individual participants' solution times using that strategy; Backup, any strategy other than retrieval; Don't know, participant was unable to give answer within the allotted time per 5,000-msec trial.

and 245.12, $p \mathrm{~s}<.001$, respectively $]$ and $2\left[F_{\mathrm{s}}(1,20)=\right.$ 210.55 and $75.82, p s<.001$, respectively]. Clearly, the identified perfectionists showed a strong tendency not to adaptively choose the more efficient retrieval strategy.

Differences between not-so-good retrievers and the other subgroups with respect to how often they could retrieve arithmetic facts are even more apparent when problem difficulty is considered. The frequency of problems for which adults could successfully use retrieval in the nochoice condition was examined as a function of problem difficulty. Small (easier) problems were defined as those with two operands having a product smaller than or equal to 25 , and large (harder) problems were defined as those with two operands having a product larger than 25 (i.e., a median split; Campbell, 1997; Campbell \& Xue, 2001).
As expected, for both experiments, retrieval was successfully used more often when the problems were easier (i.e., smaller) as opposed to harder for both addition and multiplication (all $p \mathrm{~s}<.001$ ).

Of particular interest was that reliable interactions for successful retrieval usage emerged between problem size and subgroup membership for both addition and multiplication in Experiments $1\left[F_{\mathrm{s}}(2,75)=23.43\right.$ and 93.46, $p \mathrm{~s}<.001$, respectively $]$ and $2[F \mathrm{~s}(2,82)=26.53$ and $44.19, p<.001$, respectively]. Across both experiments and operations, the good retrievers and perfectionists were able to successfully retrieve at least $98 \%$ of the presented small problems. Not-so-good retrievers used retrieval to solve, on average, approximately $88 \%$ of the smaller problems. Greater subgroup differences emerged 
Table 2

Performance of Individual Difference Groups: Mean Proportions of Correct Retrieval and Mean Performance on Individual Difference Variables

\begin{tabular}{|c|c|c|c|c|c|c|c|c|}
\hline \multirow[b]{2}{*}{ Group } & \multirow[b]{2}{*}{$n$} & \multicolumn{2}{|c|}{ Choice Allowed } & \multicolumn{2}{|c|}{ No Choice } & \multirow{2}{*}{$\begin{array}{l}\text { Working } \\
\text { Memory }\end{array}$} & \multirow{2}{*}{$\begin{array}{l}\text { Math } \\
\text { Score }\end{array}$} & \multirow[b]{2}{*}{ Digits } \\
\hline & & Add. & Mult. & Add. & Mult. & & & \\
\hline \multicolumn{9}{|l|}{ Experiment 1} \\
\hline Perfectionists & 26 & .52 & .60 & .96 & .99 & 7.8 & 101.8 & 3.4 \\
\hline Good retrievers & 36 & .88 & .92 & .97 & .96 & 8.2 & 107.7 & 3.6 \\
\hline Not-so-good retrievers & 16 & .72 & .63 & .73 & .71 & 5.8 & 94.1 & 3.1 \\
\hline \multicolumn{9}{|l|}{ Experiment 2} \\
\hline Perfectionists & 19 & .60 & .68 & .96 & .96 & 6.8 & 105.1 & 2.7 \\
\hline Good retrievers & 45 & .92 & .93 & .96 & .95 & 6.3 & 103.6 & 2.7 \\
\hline Not-so-good retrievers & 21 & .66 & .73 & .67 & .76 & 5.1 & 91.5 & 2.5 \\
\hline
\end{tabular}

Note-Math score, general math standardized score; Digits, digits named per second.

for larger problems. Across both experiments and operations, the good retrievers and perfectionists were able to successfully retrieve at least $93 \%$ of the larger problems. Not-so-good retrievers used retrieval to solve, on average, only approximately $58 \%$ of the larger problems. Thus, the not-so-good retrievers showed substantially less retrieval, particularly for larger problems.

Strategy execution. We wanted to determine whether the speed with which adults carried out memory-search and -access processes in the choice-allowed condition varied as a function of group membership. Componential analysis was used to estimate speed of memory-search and -access processes (see, e.g., Widaman et al., 1989). This was accomplished by obtaining slope values from a regression analysis, which included a predictor that captured memory strength. Mean retrieval latencies for the arithmetic problems, averaged across participants, was the outcome variable. Consistent with the SCADS, large-number problems tended to have less peaked distributions of associations than did smaller problems (see, e.g., Siegler \& Shipley, 1995). Researchers typically employ the product of the terms of the problem as the predictor of solution times (see, e.g., Campbell \& Xue, 2001; Geary \& Widaman, 1992; Hecht, 1999; LeFevre,
Sadesky, \& Bisanz, 1996; Siegler, 1988b). The slope from these regression analyses represents the estimated time in milliseconds to engage in memory-search and -access processes (Geary \& Wiley, 1991; Hecht, 1999).

Regression analyses were conducted separately for each subgroup. The dependent variable was mean solution times for retrieval trials for each problem in the choiceallowed condition. These analyses were done to determine whether subgroup differences emerged with respect to the time participants needed to carry out memory-search and -access and other peripheral processes subsumed by the intercept term. The intercepts, slopes, and variances accounted for are reported in Table 3 , segregated by individual difference subgroup and operation (addition or multiplication).

For Experiment 1, an omnibus ANOVA indicated that the time to engage in retrieval processes captured by the product slopes between groups was not significant for addition or multiplication $[F \mathrm{~s}(2,78)=1.79$ and $.66, p \mathrm{~s}>$ .10 , respectively]. This pattern of results was replicated in Experiment $2[F \mathrm{~s}(2,78)=.78$ and $.01, p \mathrm{~s}>.10$, respectively]. Planned pairwise comparisons between subgroups for both addition and multiplication revealed no significant differences between slopes in either experiment $(p \mathrm{~s}>.10)$.

Table 3

Regression Analyses (Latencies Regressed on Product) for Choice-Allowed Retrieval Trials, Segregated by Experiment, Group, and Operation

\begin{tabular}{lccccccc}
\hline \multirow{2}{*}{ Group } & \multicolumn{3}{c}{ Addition } & & \multicolumn{3}{c}{ Multiplication } \\
\cline { 2 - 3 } Good & Interc & $r^{2}$ & Slope & & Interc & $r^{2}$ & Slope \\
\hline Perfectionist & 737.8 & 42 & 5.2 & & 854.6 & 44 & 6.2 \\
Not-so-good & 758.7 & 34 & 3.3 & & 903.2 & 38 & 5.1 \\
& 872.7 & 54 & 3.2 & & $1,007.9$ & 49 & 7.2 \\
Good & 784.2 & 35 & 2.9 & & 959.9 & 40 & 6.4 \\
Perfectionist & 690.9 & 56 & 3.4 & & 922.5 & 39 & 6.7 \\
Not-so-good & 844.6 & 46 & 4.2 & & $1,057.4$ & 47 & 6.8 \\
\hline
\end{tabular}

Note-All values significant, $p<.001$. Interc stands for intercept, and is interpreted as average time in milliseconds in which students engaged in factretrieval processes that are peripheral to memory-search and -access processes. Slope is interpreted as the average time in milliseconds in which participants engaged in memory-search and -access processes. 
Thus, no differences emerged between subgroups with respect to time used to engage in memory-search and -access processes for problems that could be retrieved.

The intercept term from each regression analysis reflects the time for controlled mental processes, such as attending to and encoding the terms in the problem (Hunt, 1978; Klapp, Boches, Trabert, \& Logan, 1991), monitoring retrieval accuracy (Conway \& Engle, 1994), and verbal production (Bock, 1996; Wheeldon \& Levelt, 1995) to be carried out (Geary, Frensch, \& Wiley, 1993; Geary \& Wiley, 1991). For Experiment 1, an omnibus ANOVA revealed significant group differences in intercept terms for both addition and multiplication $[F \mathrm{~s}(2,80)=49.54$ and 19.21 , respectively, $p \mathrm{~s}<.001]$. Planned pairwise comparisons for both addition and multiplication indicated intercept differences between not-so-good retrievers and good retrievers $[F \mathrm{~S}(1,53)=86.73$ and 29.08 , respectively, $p \mathrm{~s}<.001]$. Intercept differences also emerged between not-so-good retrievers and perfectionists $\left[F_{\mathrm{s}}(1,53)=\right.$ 54.49 and 25.90, respectively, $p \mathrm{~s}<.001]$. Intercept differences did not emerge between the perfectionist and good retriever subgroups $(p s>.10)$. This pattern of results was replicated in Experiment 2 (all $p s<.001$ ), except for one pairwise comparison. That is, intercept differences did not emerge between the perfectionist and good-retriever subgroups for multiplication $[F(1,53)=.649, p>.10]$. Importantly, the results from both experiments consistently indicated that not-so-good retrievers took more time to engage in peripheral mental processes than both the good retrievers and perfectionists.

\section{Individual Difference Groups and Working Memory and Math Achievement}

The second research question addressed in this investigation was whether math-computation achievement and working memory skills of the participants were related to patterns of individual differences in strategy choices. Table 2 shows the mean performance of the adult students on both the working memory task and the math-achievement measure, segregated by group membership and experiment. Digitnaming speed did not vary as a function of individual difference group ( $p s>.10)$, and will not be considered in subsequent analyses. For Experiment 1, mean levels of total correct working memory problems were statistically different between good retrievers, not-so-good retrievers, and perfectionists $[F(2,75)=10.49, p<.001]$. There was no significant difference in memory performance between the good retrievers and the perfectionists $(p>$ .10). Planned comparisons indicated that not-so-good retrievers' mean memory performance of 5.8 items was significantly lower than that for both perfectionists and good retrievers [means $=7.8$ and $8.2 ; F(1,40)=12.81$ and $F(1,50)=21.74, p s<.001$, respectively]. This pattern of results was replicated in Experiment 2, with a significant omnibus ANOVA $[F(2,82)=4.19, p<.05]$. Significant differences emerged between both good retrievers $($ mean $=6.3)$ and perfectionists $($ mean $=6.8)$ in comparison with the not-so-good retrievers (mean $=5.1)$; $[F(1,62)=5.93$ and $F(1,38)=8.09$, respectively $]$.
Mean levels of mathematical-computation achievement (from age-based standardized scores) also differed between groups in Experiments 1 and $2[F(2,75)=8.35$, $p<.001$, and $F(2,82)=3.36, p<.05]$. No differences emerged between good retrievers and perfectionists. Planned comparisons indicated that not-so-good retrievers' mean standardized performance of 94.1 items was significantly lower than that for both good retrievers and perfectionists $[$ means $=107.7$ and $101.8 ; F(1,50)=14.40$ and $F(1,40)=5.64, p s<.05$, respectively]. An identical pattern of results emerged for Experiment 2. Both good retrievers $($ mean $=103.6)$ and perfectionists $($ mean $=$ 105.1) exhibited significantly better performance than not-so-good retrievers [mean $=91.5 ; F(1,62$ and 1,38$)=$ 5.69 and 5.41, $p \mathrm{~s}<.05]$. Not-so-good retrievers were not, as a group, math disabled, because their mean standardized scores were within a standard deviation (i.e., 15 units) of the standardization sample.

\section{DISCUSSION}

The answer to the first research question addressed by this study suggests that previous reports of multiple-strategy selection by adults reflect the presence of not-so-good retrievers and/or perfectionists in the samples. Theoretical models of simple arithmetic, such as the SCADS, will need to account for these newly identified subgroups in adults. The findings support Kirk and Ashcraft's (2001) assertion that the frequency with which adults use retrieval in choice-allowed trials does not necessarily provide a definitive perspective on how often they can retrieve the answers. In this study, some undergraduates retrieved substantially more answers in the no-choice condition than in the choice-allowed trials.

An important difference between the present subgroups and those reported in one study with children (first graders) as participants pertains to the not-so-good retrievers (Siegler, 1988a). Siegler (1988a) reported that not-sogood retrievers retrieved more often than perfectionists, but were less accurate on retrieval trials than the good retrievers. These results did not occur in the present study. That is, the present not-so-good retrievers were just as accurate as the good retrievers with respect to retrieval accuracy in the choice-allowed condition; they adaptively chose nonretrieval strategies when they were unable to retrieve the answer. Another study with first graders also reported that not-so-good retrievers chose strategies as adaptively as did the good retrievers (Kerkman \& Siegler, 1993).

SCADS should be modified to account for adults with perfectionist patterns of strategy selection in simple arithmetic. The model assumes that when a person is presented a simple arithmetic problem, he or she will use retrieval if the activation of an answer code in the distribution of associations for that problem exceeds a threshold (i.e., confidence criterion) for stating the retrieved answer (Siegler \& Shipley, 1995). Variability in the threshold of confidence required to execute retrieval has been identified in adults (Touron \& Hertzog, 2004). For perfectionists, 
the confidence criterion may be higher than necessary for maintaining accuracy (Flett \& Hewitt, 2001; Siegler, 1988a; Touron \& Hertzog, 2004). The confidence criterion may be set high by some adults because of perceived familiarity with the problem (cf. Reder \& Ritter, 1992; Yonelinas, 2001).

Consideration of rates of success for strategies is another mechanism included in the SCADS that may provide some explanation for perfectionist strategy selection. There are substantial individual differences in this construct (see, e.g., Schunn \& Reder, 2001). The SCADS includes a metacognitive system that considers a person's familiarity with the speed and accuracy of strategies used to solve a particular problem. That is, problem-specific strengths are established between each item and how successful each procedure has been for the specific problem (Siegler \& Shipley, 1995). Accordingly, perfectionists might not always access and use statistics concerning the speed and accuracy of past executions of retrieval for some simple arithmetic problems. If they did, they would use retrieval almost universally, because they are clearly able to implement retrieval on virtually every arithmetic problem.

Regarding strategy execution processes, we made comparisons between the three subgroups with respect to slopes and intercepts for choice-allowed retrieval trials. These analyses indicated that the speed with which adults engaged in memory-search and -access processes was not different between not-so-good retrievers, good retrievers, and perfectionists. Apparently, adults who tend to be unable to retrieve the answers to some problems have peaked associations between problems and answers in long-term memory for other problems that can be retrieved. These results are consistent with the idea that the speed of searching and accessing answers from long-term memory is an automatic process (Hecht, 2003) that reaches asymptote in adulthood (Geary \& Wiley, 1991; Kail, 1988; Kaye, deWinstanley, Chen, \& Bonnefil, 1989; Widaman et al., 1992). Presumably, not-so-good retrievers acquired sufficient experience solving some arithmetic problems via retrieval for memory search and access to occur at asymptotic levels of speed (Frensch \& Geary, 1993).

The present intercept differences between ability groups reflect differences in the speed of executing controlled attention-demanding processes, such as encoding and verbal production routines needed to utter the answer (Geary, Frensch, \& Wiley, 1993; Geary \& Wiley, 1991; Hecht, 2003). Ability group differences in intercept terms have been reported in research focusing on human intelligence. Specifically, intercept terms from reaction time tasks, but not slopes representing increasing task difficulty, tend to be associated with ability group differences in adult measures of IQ (see, e.g., Beauducel \& Brocke, 1993; Deary, Der, \& Ford, 2001; Jensen, 1987; Lohman, 1994). In passing, one should note that some intelligence tests include mathematical computation as part of the IQ test battery (Sattler, 1992).

Another possible explanation for differences in intercepts is that not-so-good retrievers consistently take more time to select retrieval than do the other groups. This pos- sibility seems implausible for two reasons. There is good empirical evidence that retrieval of arithmetic knowledge in adults is obligatory and automatic (LeFevre \& Kulak, 1994; Zbrodoff \& Logan, 1986). Second, the same pattern of group differences pertaining to intercept terms occurred in both the choice-allowed and no-choice conditions. No-choice trials do not require strategy selection processes (Rittle-Johnson \& Siegler, 1999; Siegler \& Lemaire, 1997). Thus, if retrieval use is based on consciously controlled strategy-selection processes, then group differences in intercept terms should be eliminated when no-choice trials are considered. To examine this possibility, mean retrieval solution times were regressed on the product predictor for forced retrieval trials (not shown in Table 3). Consistent with findings from the choiceallowed data, comparable slopes were obtained between the three subgroups for no-choice retrieval trials (slope range $=2.5-5.1$ and $2.9-8.1$ for addition and multiplication, respectively). A pattern of intercept differences for no-choice trials emerged that was consistent with the results from the choice-allowed trials. That is, the intercepts for not-so-good retrievers were significantly slower than the values obtained from the other two subgroups for both addition and multiplication. Thus, not-so-good retrievers, as a group, require more time to engage in mental processes subsumed by the intercept term, whether or not they were required to choose a strategy for determining the answer to the problem.

The second research question investigated in this study was whether subgroup differences would emerge on the counting-span measure of working memory and the standardized math-computation achievement test. Notso-good retrievers consistently showed lower mean performance on measures of working memory and general math-computation achievement. These individuals also successfully executed retrieval less often and performed attention-demanding mental processes on retrieval trials more slowly than the other subgroups. Thus, general facility in working with mathematics and numbers (see, e.g., Carroll, 1993) may explain why some adults are less proficient than others in simple arithmetic performance.

Group differences in working memory leave open the possibility, to be examined in future longitudinal research, that for not-so-good retrievers, the formation of peaked distributions of associations was disrupted for some problems by working memory constraints. The degree of strengthening may be constrained by how well the individual can simultaneously represent and process the terms (via a counting-based strategy) and the answer generated in working memory (Geary, 1993; Hecht, Torgesen, Wagner, \& Rashotte, 2001). Indeed, even successful counting itself requires substantial working memory resources (Healy \& Nairne, 1985). Consistent with this possibility, math-disabled children tend to have lower working memory and to choose retrieval less often than normally achieving peers (Geary, 1993). Research with adults does suggest that memory constraints influence the rate of formation of associations between problems and answers in long-term memory (Klapp et al., 1991). 


\section{Conclusion}

It is clear from the present results that concurrent use of the choice/no-choice method and verbal protocols provides data that can be used to form rationally defined subgroups of individuals. Of particular importance is that the no-choice condition enabled an accurate estimate of the frequency with which adults can use retrieval to solve arithmetic problems. The findings put in high relief the importance of distinguishing between strategies that adults freely choose and strategies that adults successfully implement when not given a choice. Future updates of the SCADS will need to accommodate characteristics of adults (cf. Smith-Chant \& LeFevre, 2003), including notso-good-retriever and perfectionist patterns of strategy selection.

\section{REFERENCES}

AshCraft, M. (1995). Cognitive psychology and simple arithmetic: A review and summary of new directions. Mathematical Cognition, $\mathbf{1}$, 3-34.

BADDELEy, A. D. (1992). Is working memory working? The Fifteenth Bartlett Lecture. Quarterly Journal of Experimental Psychology, 44A, $1-31$.

BeAuducel, A., \& BRocke, B. (1993). Intelligence and speed of information processing: Further results and questions on Hick's paradigm and beyond. Personality \& Individual Differences, 15, 627-636.

BосK, K. (1996). Language production: Methods and methodologies. Psychonomic Bulletin \& Review, 3, 395-421.

CAMPBELL, J. I. D. (1997). On the relation between skilled performance of simple division and multiplication. Journal of Experimental Psychology: Learning, Memory, \& Cognition, 23, 1130-1159.

Campbell, J. I. D., \& Xue, Q. (2001). Cognitive arithmetic across cultures. Journal of Experimental Psychology: General, 130, 299-315.

CARroll, J. B. (1993). Human cognitive abilities: A survey of factoranalytic studies. New York: Cambridge University Press.

Conway, A. R. A., \& ENGLE, R. W. (1994). Working memory and retrieval: A resource-dependent inhibition model. Journal of Experimental Psychology: General, 123, 354-373.

Deary, I. J., Der, G., \& Ford, G. (2001). Reaction times and intelligence differences: A population-based cohort study. Intelligence, 29, 389-399.

Flett, G. L., \& Hewitt, P. L. (2001). Perfectionism: Theory and research. Washington DC: American Psychological Association.

Frensch, P. A., \& Geary, D. C. (1993). Effects of practice on component processes in complex mental addition. Journal of Experimental Psychology: Learning, Memory, \& Cognition, 19, 433-456.

GaGNE, R. M. (1983). Some issues in the psychology of mathematics instruction. Journal for Research in Mathematics Education, 14, 275282.

Geary, D. C. (1993). Mathematical disabilities: Cognitive, neuropsychological, and genetic components. Psychological Bulletin, 114, 345-362.

Geary, D. C., Frensch, P. A., \& Wiley, J. G. (1993). Simple and complex mental subtraction: Strategy choice and speed-of-processing differences in younger and older adults. Psychology \& Aging, 8, 242-256.

Geary, D. C., \& Widaman, K. F. (1992). Numerical cognition: On the convergence of componential and psychometric models. Intelligence, 16, 47-80.

GearY, D. C., \& Wiley, J. G. (1991). Cognitive addition: Strategy choice and speed-of-processing differences in young and elderly adults. Psychology \& Aging, 6, 474-483.

Goldman, S. R., Mertz, D. L., \& Pellegrino, J. W. (1989). Individual differences in extended practice functions and solution strategies for basic addition facts. Journal of Educational Psychology, 81, 481-496.

Healy, A. F., \& NaIRNE, J. S. (1985). Short-term memory processes in counting. Cognitive Psychology, 17, 417-444.
Hecht, S. A. (1998). Toward an information-processing account of individual differences in fraction skills. Journal of Educational Psychology, 90, 545-559.

Hecht, S. A. (1999). Individual solution processes while solving addition and multiplication math facts in adults. Memory \& Cognition, 27, 1097-1107.

HeCHT, S. A. (2002). Counting on working memory in simple arithmetic when counting is used for problem solving. Memory \& Cognition, 30, 447-455.

HeChT, S. A. (2003). Sources of individual differences in fraction skills. Journal of Experimental Child Psychology, 86, 277-302.

Hecht, S. A., Torgesen, J. K., Wagner, R. K., \& Rashotte, C. A. (2001). The relations between phonological processing abilities and emerging individual differences in mathematical computation skills: A longitudinal study from second to fifth grades. Journal of Experimental Child Psychology, 79, 192-227.

Hitch, G. J., \& MCAULEY, E. (1991). Working memory in children with specific arithmetical learning difficulties. British Journal of Psychology, 82, 375-386.

Hunt, E. (1978). Mechanics of verbal ability. Psychological Review, $\mathbf{8 5}, 109-130$.

Jensen, A. R. (1987). Individual differences in the Hick paradigm. In P. A. Vernon (Ed.), Speed of information-processing and intelligence (pp. 101-175). Norwood, NJ: Ablex.

Jordan, N. C., Hanich, L. B., \& Kaplan, D. (2003). Arithmetic fact mastery in young children: A longitudinal investigation. Journal of Experimental Child Psychology, 85, 103-119.

KaIL, R. (1988). Developmental functions for speeds of cognitive processes. Journal of Experimental Child Psychology, 45, 339-364.

Kaye, D. B., DeWinstanley, P., Chen, Q., \& Bonnefil, V. (1989). Development of efficient arithmetic computation. Journal of Educational Psychology, 81, 467-480.

Kerkman, D. D., \& Siegler, R. S. (1993). Individual differences and adaptive flexibility in lower-income children's strategy choices. Learning \& Individual Differences, 5, 113-136.

Kirk, E. P., \& AshCraft, M. H. (2001). Telling stories: The perils and promise of using verbal reports to study math strategies. Journal of Experimental Psychology: Learning, Memory, \& Cognition, 27, 157175.

Klapp, S. T., Boches, C. A., Trabert, M. L., \& Logan, G. D. (1991). Automatizing alphabet arithmetic II: Are there practice effects after automaticity is achieved? Journal of Experimental Psychology: Learning, Memory, \& Cognition, 17, 196-209.

LeFevre, J.-A., Bisanz, J., Daley, K. E., Buffone, L., Greenham, S. L., \& SADESKY, G. S. (1996). Multiple routes to solution of singledigit multiplication problems. Journal of Experimental Psychology: General, 125, 284-306.

LeFevre, J.-A., \& KulaK, A. G. (1994). Individual differences in the obligatory activation of addition facts. Memory \& Cognition, 22, 188200.

LeFevre, J.[-A.], Sadesky, G. S., \& Bisanz, J. (1996). Selection of procedures in mental addition: Reassessing the problem size effect in adults. Journal of Experimental Psychology: Learning, Memory, \& Cognition, 22, 216-230.

LoHman, D. F. (1994). Component scores as residual variation: or Why the intercept correlates best. Intelligence, 19, 1-11.

Reder, L. M., \& Ritter, F. E. (1992). What determines initial feeling of knowing? Familiarity with question terms, not with the answer. Journal of Experimental Psychology: Learning, Memory, \& Cognition, 18, 435-451.

RitTle-Johnson, B., \& Siegler, R. S. (1999). Learning to spell: Variability, choice, and change in children's strategy use. Child Development, 70, 332-348.

Sattler, J. M. (1992). Assessment of children (3rd ed.). San Diego: J. M. Sattler.

SchunN, C. D., \& Reder, L. M. (2001). Another source of individual differences: Strategy adaptivity to changing rates of success. Journal of Experimental Psychology: General, 130, 59-76.

Shrager, J., \& Siegler, R. S. (1998). SCADS: A model of children's strategy choices and strategy discoveries. Psychological Science, 9, 405-410. 
SIEGLER, R. S. (1988a). Individual differences in strategy choices: Good students, not-so-good students, and perfectionists. Child Development, 59, 833-851.

SIEGLER, R. S. (1988b). Strategy choice procedures and the development of multiplication skill. Journal of Experimental Psychology: General, 117, 258-275.

Siegler, R. S., \& Lemaire, P. (1997). Older and younger adults' strategy choices in multiplication: Testing predictions of SCADS using the choice/no-choice method. Journal of Experimental Psychology: General, 126, 71-92.

Siegler, R. S., \& Shipley, E. (1995). Variation, selection, and cognitive change. In G. Halford \& T. Simon (Eds.), Developing cognitive competence: New approaches to process modeling (pp. 31-76). Hillsdale, NJ: Erlbaum.

Smith-Chant, B. L., \& LeFevre, J.-A. (2003). Doing as they are told and telling it like it is: Self-reports in mental arithmetic. Memory \& Cognition, 31, 516-528.

Touron, D. R., \& Hertzog, C. (2004). Strategy shift affordance and strategy choice in young and older adults. Memory \& Cognition, 32, 298-310.

Wagner, R. K., Torgesen, J. K., \& Rashotte, C. A. (1994). Development of reading-related phonological processing abilities: New evidence of bidirectional causality from a latent variable longitudinal study. Developmental Psychology, 30, 73-87.
Wheeldon, L. R., \& Levelt, W. J. M. (1995). Monitoring the time course of phonological encoding. Journal of Memory \& Language, 34, 311-334.

Widaman, K. F., Geary, D. C., Cormier, P., \& Little, T. D. (1989). A componential model for mental addition. Journal of Experimental Psychology: Learning, Memory, \& Cognition, 15, 898-919.

Widaman, K. F., Little, T. D., Geary, D. C., \& Cormier, P. (1992). Individual differences in the development of skill in mental addition: Internal and external validation of chronometric models. Learning \& Individual Differences, 4, 167-213.

Woodcock, R. W., \& Johnson, M. B. (1989). Woodcock-Johnson Test of Achievement Standard Battery, Form B. Itasca, IL: Riverside.

Yonelinas, A. P. (2001). Consciousness, control, and confidence: The 3 Cs of recognition memory. Journal of Experimental Psychology: General, 130, 361-379.

ZBrodoff, N. J., \& LogAN, G. D. (1986). On the autonomy of mental processes: A case study of arithmetic. Journal of Experimental Psychology: General, 115, 118-130.

(Manuscript received July 27, 2004; revision accepted for publication January 19, 2005.) 